\title{
Avoiding the pitfalls of sponsored multicentre research in general practice
}

\author{
Heather A Waldron, Ronald F Cookson
}

\begin{abstract}
Research in general practice is becoming increasingly popular, and most general practitioners will sooner or later have to decide whether to become involved with clinical trials sponsored by drug companies. This paper outlines the advantages and disadvantages of multicentre research-based on experience of running a research group since the early $1980 \mathrm{~s}-$ to enable doctors to reach the appropriate decision and to avoid involvement in trials which are either unethical or ineffective.
\end{abstract}

\section{Do we need multicentre trials in general practice?}

Trials in general practice are becoming increasingly popular $^{1}$ and have many attractions. They offer the opportunity for rapid collection of data from large populations of patients and provide results that are relevant to clinical practice in a primary care setting. ${ }^{2}$ In contrast, hospital studies tend to focus on referred or seriously ill patients in a controlled environment and so are of limited value in community medicine.

Multicentre trials permit centres to be included that by themselves would have too small a population for a trial. ${ }^{34}$ Collaboration with other doctors ensures that sufficient patients are recruited in a reasonable time and offers the potential for learning from the experience of others.

From the general practitioner's point of view, multicentre trials provide the only realistic opportunity to become involved in major research without a disproportionate investment of time and money; they can also be a good school to learn research. In a sponsored trial, the general practitioner has the advantage of leaving

Research and Development Department, Janssen Pharmaceutical Limited, Wantage OX12 ODQ Heather A Waldron, medical development manager Ronald F Cookson, director of medical development and information

Correspondence to: Dr Cookson.

BMY 1993;307:1331-4

without a trial.

- Efficacy claims are sometimes made when a safety statement may be the only valid conclusion.

- Important side effects, although mentioned in tables and results, may receive less attention in conclusions-unless they confer advantage on the sponsor's drug.

- Studies often do not include a placebo.

- Relatively small populations are compared without any discussion of the power of the study to distinguish statistically significant differences between them.

- There may be no mention of ethics approval or written informed consent, two absolute requirements of any clinical trial.

- The identity of the sponsor, or even the country in which a study is performed, is not always clear. Contract clinical research organisations can publish research carried out on behalf of other companies without revealing such information; investigators and publishers should be more discriminating. the logistics and financial planning to others but the disadvantage of negotiating or accepting what is offered, often on a take it or leave it basis.

The British system of family doctors ensures that general practice studies in this country are attractive. The American Food and Drug Administration will accept data from British trials in general practice conducted to the appropriate standard; hence the surge in interest in this area in the past few years, particularly from contract research organisations working for large Japanese or American pharmaceutical companies. The identity of the trial sponsor has important implications since overseas companies are not necessarily subject to the Association of the British Pharmaceutical Industry's codes of practice, which govern the behaviour of pharmaceutical companies in the United Kingdom. ${ }^{5}$ Doctors should therefore always check the identity of the trial sponsor. Participants in clinical trials (both doctors and patients) also need to be aware that not every drug studied in Britain is destined for the British market.

\section{How is clinical research regulated?}

All phase IV trials (those involving licensed products in licensed indications) sponsored by pharmaceutical companies in the United Kingdom should conform to the Association of the British Pharmaceutical Industry's Code of Practice for the Clinical Assessment of Licensed Medicinal Products in General Practice most recently revised in March $1992 .{ }^{6}$ Preregistration (phase II and III) trials are regulated by various guidelines on good clinical research practice. ${ }^{7}$ The guidelines on good clinical research practice have practical implications for doctors involved in trials; for instance, doctors are now required to keep copies of patients' record forms for 15 years after the trial, and they may be involved in more paper work and monitoring visits than before to fulfil stringent auditing requirements.
Do multicentre general practice trials produce high quality, reliable data?

Recent papers arising from multicentre research by general practitioners sponsored by industry and by academic units underline the need for clear, scientific and ethically sound protocols; properly conducted studies; appropriate statistical analysis; and carefully written papers. Publications are often open to criticism on the grounds that they throw little light on methodology and particularly for the way exaggerated claims may be made in the guise of scientific analysis. Other problems we have encountered are listed in box 1 .

Despite the problems, much good research is done in general practice and, in our experience, the main problem has been not the quality of data produced but the quantity of it. Doctors tend to overestimate the number of patients they can enrol into a trial, and the 
recruitment rate is therefore often slower than the organisers had hoped for. ${ }^{8-11}$ One example of this is the study reported by Tognani $e t a l$, in which 806 general practitioners agreed to participate but only 63 actually recruited and followed up at least one patient. ${ }^{8}$

One way to avoid this problem is for the organiser to take advice from experienced investigators on achievable recruitment rates. The rate can then be validated by supplying a few doctors with the planned criteria for inclusion or exclusion in the trial and asking them to record all eligible patients seen during a pilot pretrial period. The resulting rate should then be halved to give a realistic estimate for the recruitment period that the study will require. Even with such estimates, patient recruitment may be lower than predicted. The figure shows actual and expected patient recruitment from an unpublished gastroenterology trial-rates are reasonable in the first few months and tail off as initial enthusiasm wanes.

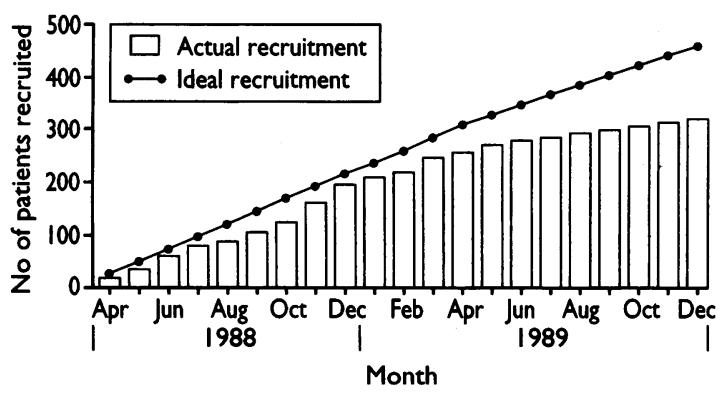

\section{How do trials affect the patient?}

The nature of the relationship between general practitioners and patients provides both advantages and potential problems in clinical trials. Participating doctors need to bear in mind that they will be responsible for obtaining the patient's written, informed consent and must consider how to explain concepts such as use of placebo and treatment randomisation in a clinical setting. Patients may believe that their doctor's suggestion that they enter a trial is a recommendation that this is the best course of action for them; this should be remembered when ensuring that properly informed consent is given. Patients may agree to take part in a study because they believe they would face disapproval or even not receive as much attention from their doctor if they refused. Patients take on trust a great deal that the doctor may also have assumed but not necessarily checked. ${ }^{12}$

To assist general practitioners in explaining trials to patients, organisers should produce an information leaflet to give to patients considering taking part in a trial. This should explain why trials are necessary and set out the principles of using a placebo or comparing two active treatments in a double blind fashion. It should also point out that participating in a trial may involve filling in diary cards or having extra tests performed.

Both the doctor and the patient should be clear about treatment options after the trial has ended. This is not usually a problem in trials of products licensed in the United Kingdom because if the patient responds well to treatment during the trial the doctor can prescribe the drug in the usual way. Continuing with the preferred treatment may, however, be difficult if the patient has been receiving placebo or an active but unlicensed drug.

In multicentre, double blind trials there may also be problems of discovering what treatment the patient has been receiving. Codes are not broken until the trial data have been collected from the last patient in the study. This may be many months after the treatment regimen for earlier participants has stopped. Some manufacturers make provision for the blind continuation of treatment until codes are broken.

The increasing emphasis on indicative prescribing and general practitioner budgets may also generate issues of cost when expensive treatments cease to be provided free by the manufacturer at the end of a trial. The policy for product availability after the trial has ended should be stated in the protocol.

\section{How do trials affect general practitioners?}

Although, in theory, research should mirror clinical practice and involve a minimum of extra work, ${ }^{4}$ in reality, with the arrival of good clinical research practice, an additional time commitment is required. Gone are the days when a group of family doctors could write that carrying out research "provided a pleasant and educational diversion from the normal office routine."13

The main problem is pressure of time; the paper work can be tedious, and the extra time spent with the patient can disrupt the surgery routine. On the other hand, the extra time allocated to patients may be welcomed.

One way of lessening the paperwork burden is to involve a practice nurse. The organisers may provide training for practice nurses to go through patient record forms to make sure they are properly completed before the monitoring visits. This spares the doctors from chasing basic demographic data and saves time at the monitoring visit.

Good clinical research practice requires close monitoring of trials by clinical research associates employed by the sponsor or a contract research organisation. They can provide help to the investigator, and they provide an important link with the organising company. However, doctors should be prepared to make time for monitoring visits, and they need to remember that monitors are required to check patient records. To preserve patient confidentiality, "back to back" checking may be used. This involves the doctor consulting the patient's records to answer the monitor's questions about details such as patient's date of birth, NHS number, and dates of appointments. Some doctors prefer to inform their patients that monitors may need to see their notes, and make agreement for this a condition for entry to a trial.

\section{What to look for in a sponsored clinical trial THE PROTOCOL}

The protocol should identify the sponsor and describe the experiment (that is, the questions to be answered) and how the study will be performed; a written copy, signed by both parties, should be provided to the general practitioner by the sponsor. The protocol is a contract, with legal implications, between the doctor and the sponsor, and should be treated as such. Although doctors may not yet feel threatened with breach of contract if they do not put in appropriate patients and do not exercise due diligence in completing the forms, increasingly they are paid only for usable data.

\section{PAYMENT}

While it is reasonable for doctors to expect reimbursement for the additional time spent on the trial, the line between reimbursement for effort and undue incentive to put a patient on a particular drug is easy to cross and doctors should consider potential conflicts of interest or influences on their practice arising from payment for studies. Many ethics committees now require a payment schedule to be attached to the protocol for approval. ${ }^{14}$ The BMA issues recommended rates which many sponsors use, but the 
increasing number of trials required for product registration has led to a significant escalation in the market rates for experienced general practitioner researchers. The current hourly rate recommended by the BMA for participation in clinical trials is $£ 109.50$, whereas in the early 1980 s the rate was $£ 60 .{ }^{15}$ As a rule of thumb, participants might consider whether they would be happy to inform their patients about financial arrangements for a trial.

ETHICAL APPROVAL

Most multicentre trials are approved by a central, independent ethics committee. It is the general practitioner's responsibility to obtain additional local ethical approval as required. When ethics committees of standing, such as the Royal College of General Practitioners clinical trials ethics committee, are quoted, studies tend to be of more value. ${ }^{16} 17$

General practitioners should also remember that local ethics committees may be helpful if they have concerns about a protocol or want the benefit of an experienced second opinion in deciding whether a trial is worthwhile or a protocol sound. Formal approval may take a little while, but informal advice can usually be obtained quite quickly.

\section{TRAINING}

It is essential for multicentre studies that all investigators are trained to use the same diagnostic methodology and outcome measures to reduce interinvestigator variability. Patient selection criteria must be definite and consistent, and all participants should work together to obtain as uniform a study population as possible. Training in diagnosis and the opportunity to work with doctors from other regions may be an incentive for general practitioners to become involved with trials.

Simple procedures such as patient visual analogue symptom scores or diaries are probably self explanatory, but the use of complex rating scales (such as those found in psychiatry) or technically complex measurements (cardiovascular, for instance) often requires special training. Sponsors may run study sessions for investigators on diagnostic and assessment procedures and may offer research training, which is often a neglected part of medical education. ${ }^{18}$

Explanation of criteria for entry into the trial is particularly important. If these are not clearly understood by the doctor then patients may be dropped from the trial after randomisation when checking by the monitor reveals that ineligible patients have been entered. Many trials now underline the importance of checking eligibility by paying doctors only for usable data from eligible patients.

AUDIT

Audits are needed to ensure that trials are carried out according to the protocol and to the standards of good clinical research practice. The current British guidelines require on site monitoring and audit of a percentage of the patient record forms. In considering the time required for a study, doctors should not forget the need for regular monitoring visits and audit.

The sponsor should provide well qualified study monitors to check and validate the data and should monitor investigators and their progress. The Association of the British Pharmaceutical Industry recommends that company medical departments should take sole responsibility for the organisation of clinical trials to ensure that the distinction between research and marketing does not get blurred. ${ }^{67}$

FEEDBACK AND PUBLICATION

Doctors should inquire about arrangements for keeping them regularly informed of the progress of the trial; this may take the form of written reports, monitors' visits, and investigators' meetings. Meetings are not universally popular, ${ }^{19}$ but without them investigators remain isolated and have little influence on the trial. They also offer an opportunity for education and the sharing of experience.

Investigators should also find out the sponsor's plans for communicating the final results. All participants should receive a final research report and have an opportunity to comment on the analysis and conclusions. Local ethics committees may also require a report from their investigator. Policies for wider publication should also be clearly set out. Patients might reasonably expect that their contribution to a trial will not languish in a computer or filing cabinet nor be used inappropriately, and the doctor has a certain responsibility in ensuring that this is the case and checking with the sponsors if problems arise. Sponsors are usually keen to encourage the publication of favourable results but may be less enthusiastic if the results are disappointing to them. If investigators initiate publications themselves they are usually required to consult the sponsor before submitting an article.

Authorship and acknowledgement policies for published papers should be agreed in advance. One problem of large multicentre trials, whether sponsored or independent, is that no single investigator will have sufficient data for publication. Therefore it should be agreed in advance that one or two principal investigators will have access to all the data and be named as the main authors in publications. This should reduce the likelihood of multiple publication by several investigators and make clear who is responsible for publication. Named authorship is usually reserved for investigators who have been involved in designing the protocol, analysing the data, and drafting the paper. Other investigators may be acknowledged, either as a group or individually as appropriate.

\section{Joining a research group}

Many of the potential pitfalls of sponsored multicentre trials in general practice can be avoided by joining a formal research group such as the one described in box 2 . Such a group facilitates training, communication, and mutual trust (both between the sponsor and the doctors and among the investigators)

\section{Box 2-Janssen Research Group}

In 1981 Janssen Pharmaceutical Ltd set up a research group of general practitioners known as the Janssen Research Group. The initial group of 20 has grown to over 100. A formal written constitution outlines the objectives and rules of the group. The executive committee comprises three general practitioners and three company staff. Meetings and newsletters are organised by Janssen staff.

Protocols are written by experienced medical department staff in consultation with experts in toxicology, pharmacy, pharmacology, or pharmacokinetics. Early input from a statistician plays an important part in study design. Trial protocols and record forms are critically reviewed by specialised committees of general practitioners who advise on their practicality and design before they are finalised. Members are also consulted on inclusion and exclusion criteria, likely recruitment rates, and how much of the general practitioner's time a trial will take.

Meetings are held at the start of trials and then at least every six months to keep investigators motivated and involved. Doctors receive monthly recruitment updates for trials in which they are taking part and also a quarterly newsletter. At the end of the trial all investigators receive a full research report and the results are presented at group meetings. 


\section{Box 3-Checklist for doctors considering taking part in a trial}

- Is the trial's objective clearly stated?

- Is the trial's objective scientifically relevant?

- Is the design appropriate, and are patient numbers

sufficient to provide a valid answer?

- Who is running and sponsoring the trial?

- What are the legal liability and drug supply arrangements, before and after the trial?

- How is the patient protected in the trial?

- Has ethical approval been obtained? (Check requirements for local ethics committees.)

- Is patients' written, informed consent required?

- How much time will the trial take (include monitoring visits)?

- Is the proposed payment reasonable (and not excessive)?

- Who will monitor the trial and how often?

- What feedback will you receive during the trial?

- What will happen to the data at the end of the trial, and what is the publication policy?

Belonging to a group enables doctors to be involved with protocol development and practical issues such as design of the record form and reduces the feeling that involvement in the trial is on a take it or leave it basis. Group identity may also reduce the temptation of duplicate publication while ensuring that key investigators have access to the complete results and can therefore initiate publications themselves. Participants also enjoy the regular contact with other general practitioners sharing a common research interest.

\section{Conclusions}

Box 3 provides a list of questions to consider before agreeing to take part in a multicentre, sponsored trial. If doctors are unsure about any of these points they should seek advice from a third party with experience of assessing protocols (their local ethics committee, for example) and, where appropriate, the trial's sponsor. Only when the general practitioner has a satisfactory reply to each of the questions should he or she agree to participate. Simply asking these questions should reduce the number of inappropriate drug trials in the future.

We thank Drs Mike Emanuel and Peter Tooley, as well as members of the Janssen Research Group, for comment and advice over the past 10 years. We also thank Elizabeth Healing for help with this manuscript. Copies of a standard trial information leaflet and the constitution of the Janssen Research Group are available on request from the authors.

1 Silagy $C$. Developing a register of randomised controlled trials in primary care. BMF 1993;306:897-900.

2 Greenberg G. Clinical trials in general practice. BMf 1991;303:940.

3 Áberg H. Multicentre trials in general practice. Scand 7 Prim Health Care 1989;7:129-30.

4 Warlow C. How to organise a multicentre trial. $B M F 1990 ; 300: 180-3$.

5 Feinmann J. Look out for unethical drug trials. General Practitioner 1990;Sep 28:40.

6 Association of the British Pharmaceutical Industry. Code of practice for the clinical assessment of licensed medicinal products in general practice. London: ABPI, 1992.

7 Association of the British Pharmaceutical Industry. Guidelines on good clinical research practice. London: ABPI, 1988.

8 Tognoni G. Alli C, Avanzini F, Bettelli G, Colombo F, Corso R, et al. Randomised clinical trials in general practice: lessons from a failure. $B M \mathscr{f}$ 1991;303:969-71.

9 Peto V, Coulter A. Randomised clinical trials in clinical practice. $B M \mathcal{F}$ 1991;303:1549-50.

10 Bailey BJ. Cooperative clinical research in otology. Am f Otol 1989;10:248-51.

11 Jonker PL, Sumajow CA. Randomised clinical trials in general practice. $B M \mathcal{F}$ 1992;304:508.

12 Maj M. Multicentre psychopharmacological trials some guidelines on methodological and ethical issues. Prog Neuropsychopharmacol Biol Psychiatry 1989;13:ix-xiii.

13 Bope ET, Adams RC, Brewer SC, Cairns CB, Fosnaugh NR, Leidheiser PC et al. Multicenter private practice comparison of tripamide and hydrochlor thiazide in the treatment of hypertension. Fam Pract Res $f 1$ 1988;7:197-204.

14 Royal College of Physicians. Guidelines on the practice of ethics committees in medical research involving human subjects. London: RCP, 1990.

15 BMA announces category D fees. General Practitioner 1993 March 26:72.

16 Bingham K. Why clinical research is worth the trial for GPs. Doctor 1991;February 14:29.

17 Philip IG, Qureshi SM, Richards HH, Sharma SK, Wright FG, Young PH, et al. A comparison of a hydrochlorthiazide combination (Dyazide) and atenolol in the treatment of patients with mild hypertension: a multicentre study in general practice. Br f Clin Pract 1987;41:947-53.

18 Gray DP. Research in general practice: law of inverse opportunity. $B M F$ 1991;302:1380-2.

19 Marley JE, Snaith J. Does communication between investigators improve the conduct of the multicentre study? $\mathrm{Br}$ f Clin Pract 1989;43:158-60.

(Accepted 12 August 1993)

\section{WORK EXPERIENCE}

\section{To be a doctor?}

I arrived at the hospital clutching the scrap of paper on which was written the name of the houseman I was to shadow for the next four days.

I am 17 and in the lower sixth form at school. I hope to become a doctor, but several housemen have told me that if they had known what the hours were like beforehand they would have thought twice. Housemen seem to get the worst deal, and I arranged the work experience to get an idea of what their life is like.

I found that there is a lot of on call and little chance for relaxation. It is not romantic when the bleep goes at two in the morning and you have to get up to see a drunk person in casualty. The excitement and tension shown on television programme like Casualty are rare.

In many ways, though, it was inspiring. The doctors were devoted to their patients. They were kind, tolerant, and understanding, however cynical they might seem behind the scenes. Several times in response to the statement, "I hope to do medicine," they told me, "Don't Do law."

I found that no matter how much you read, think, or talk about medicine, nothing can replace being in a hospital. I had no idea how I would react to someone dying in front of me until it happened. Three of our patients died during the time I was in the hospital, all with cardiac arrests. On the first occasion the technology partly cut me off from the human side of death. There were lots of people crowding round, frantically doing things, then they stopped after they agreed that the patient was dead. But the patient didn't look any different; nothing had changed except that now everything was still. I found it hard to equate what had happened with an intense and personal loss for someone.

The second time it was easier to think about the person behind the machines. From a doctor's point of view, people die all the time. I think it could be hard to remember that each death can never happen again and that each person's grief is different and cannot just be given a prepackaged response. While it is important to respond with sympathy and respect you must not take every death personally and must keep it in proportion.

I enjoyed the four days so much that I didn't want to go home. Spending that time in the hospital answered several fundamental questions I had and made me absolutely sure that I would like to be a doctor.

Work experience in a hospital is certainly worth while to a potential medical student. A careers adviser had told me that it was pointless applying to consultants because they didn't like work experience, but the one I met could not have been kinder.-JENNY RUSHTON is a schoolgirl in Sale

We are delighted to receive submissions of up to 600 words on $A$ paper (or patient or book) that changed my practice, $A$ memorable patient, The one message I would like to leave behind, or related topics. 